\title{
Conhecimento de puérperas sobre identificação e prevenção do acidente loxoscélico
}

\author{
Puerperal women's knowledge on the identification and prevention of loxoscelic accidents \\ Conocimiento de las puérperas sobre la identificación y prevención de accidentes loxoscélicos
}

Guilherme Luiz Pacher Schmitz. Universidade Positivo (UP). Curitiba, PR, Brasil. guilhermelpschmitz@ hotmail.com

Ignatz Rohrbacher. Universidade Positivo (UP). Curitiba, PR, Brasil. ignatz.rohrbacher@gmail.com. (Autor correspondente)

Carlos Júnio da Silva Corrêa. Universidade Positivo (UP). Curitiba, PR, Brasil. carlos_junio100 @ hotmail.com

Aglaher Mayra Stocco da Silva. Universidade Positivo (UP). Curitiba, PR, Brasil. aglaherstocco@yahoo.com.br

Cerlei Fátima Franzoi. Universidade Positivo (UP). Curitiba, PR, Brasil. cfranzoi@ onda.com.br

Faigha Barbosa Parzianello. Universidade Positivo (UP). Curitiba, PR, Brasil. faighaa@ hotmail.com

Jarbas Pereira Silva. Universidade Positivo (UP). Curitiba, PR, Brasil. jar_bass@yahoo.com.br

Felipe Franchini Rezende. Universidade Positivo (UP). Curitiba, PR, Brasil. felipefranchini@ hotmail.com

Carlos Frederico Oldenburg Neto. Universidade Positivo (UP). Curitiba, PR, Brasil. coldenburg@uol.com.br

\section{Resumo}

Objetivos: este estudo teve como objetivo identificar o grau de conhecimento que puérperas possuíam a respeito da aranha Loxosceles, sua picada e conduta no caso de acidente com elas ou seus filhos. Métodos: estudo transversal quantitativo, que utilizou um questionário estruturado abrangendo perfil socioeconômico, conhecimento sobre aranha-marrom e o loxoscelismo. Foram incluídas pacientes em puerpério imediato, considerado entre o primeiro e décimo dia do parto, internadas na maternidade do Hospital do Trabalhador em Curitiba-PR, entre julho e outubro de 2011. Participaram 208 puérperas, das quais oito foram excluídas porque os questionários foram preenchidos de forma incorreta. Os dados obtidos foram tabulados no programa Microsoft Excel $2007^{\circledR}$ e analisados pelos testes $\chi^{2} \mathrm{e}$ Exato de Fisher. Resultados: sobre o conhecimento da aranha, $61 \%$ das puérperas afirmaram reconhecer a aranha e apontaram a imagem correta. Sobre a conduta em caso de acidente, $87,5 \%$ responderam a conduta correta, porém apenas $37 \%$ apontaram a sintomatologia correta. Hipóteses testadas mostraram que idade, escolaridade e acidente loxoscélico prévio são diretamente proporcionais ao conhecimento das participantes $(p<0,05)$. Conclusão: a maioria das puérperas pesquisadas conhecia a aranha-marrom e grande parte reconhecia a lesão provocada pela picada, mas a gravidade da lesão foi superestimada. Contraditoriamente, o conhecimento dos sintomas foi pequeno, o que pode levar ao atraso do tratamento delas mesmas ou dos seus filhos. O conhecimento da aranha foi diretamente relacionado à idade, escolaridade e a acidente loxoscélico prévio.

\begin{abstract}
Objectives: this study aimed to identify puerperal women's level of knowledge about the Loxosceles spider, its bite, and their own conduct in the event of a loxoscelic accident involving them or their children. Methods: this was a quantitative cross-sectional study using a structured questionnaire that included the socioeconomic profile of the participants, and knowledge about the brown spider and loxoscelism. Participants included postpartum women (between the first to tenth day of delivery), admitted to the maternity ward of the Hospital do Trabalhador, Curitiba-PR, between July and October 2011. From a total of 208 postpartum women, eight were excluded because the questionnaires were filled incorrectly. Data were tabulated using Microsoft Excel $2007^{\circledR}$ and analyzed using the $\chi^{2}$ test and Fisher's exact test. Results: regarding the knowledge about the spider, $61 \%$ of the participants said that they knew the spider and identified the correct picture. About their conduct in case of loxoscelic accidents, $87.5 \%$ gave appropriate responses, but only $37 \%$ identified the correct symptoms. Hypothesis testing revealed that age, education, and prior experience of loxoscelic accidents were directly proportional to the participants' knowledge $(p<0.05)$. Conclusion: most of the women surveyed could identify the brown spider and largely recognized the spider's bite injury, but they overestimated its severity. On the contrary, they had little knowledge about the symptoms, which may lead to delayed treatment for them or their children. The participants' knowledge about the spider was directly related to age, education, and prior experience of a loxoscelic accident.
\end{abstract}

Como citar: Schmitz GLP, Rohrbacher I, Corrêa CJS, Silva MAS, Franzoi CF, Parzianello FB, Silva JP, Rezende FF, Oldenburg Neto CF. Conhecimento de parturientes sobre identificação e prevenção do acidente loxoscélico. 2015;10(36)1-8. http://dx.doi.org/10.5712/rbmfc10(36)778
Palavras-chave:

Aracnidismo

Mordeduras e Picadas

de Insetos

Saúde Pública

Prevenção de acidentes

Educação em Saúde

Keywords:

Arachnidism

Insect Bites and Stings

Public Health

Accident Prevention

Health Education

Fonte de financiamento: declaram não haver. Parecer CEP:

345/2011 (SESA/HT).

Conflito de interesses: declaram não haver. Procedência e revisão por pares: revisado por pares.

Recebido em: 08/07/2013.

Aprovado em: 06/03/2014. 


\section{Resumen}

Objetivos: este estudio tuvo como objetivo identificar el grado de conocimiento que las puérperas tenían respeto la araña Loxosceles, su picadura y la conducta en el caso de un accidente con ellas o sus hijos. Métodos: estudio transversal cuantitativo en que se utilizó un cuestionario estructurado con el perfil socio-económico, el conocimiento sobre la araña marrón y el Loxoscelismo. Las pacientes incluidas fueron aquellas en el período inmediatamente posterior al parto, del primero hasta el décimo día puerperal, admitidas en la Maternidad y Hospital del Trabajador en Curitiba- PR, entre julio y octubre del 2011. Participaron 208 puérperas, de las cuales ocho fueron prohibidas debido a que sus cuestionarios fueron completados incorrectamente. Los datos fueron tabulados en Microsoft ${ }^{\circledR}$ Excel 2007 y analizados mediante el Test de $\chi^{2}$ y Exacto de Fisher. Resultados: respeto al conocimiento de la araña, el $61 \%$ de las madres dijeron reconocer la araña y apuntaron a la imagen correcta. Acerca de la conducta en caso de accidentes, el 87,5\% respondieron la conducta correcta, pero sólo el $37 \%$ identificaron los síntomas correctos. Las hipótesis testadas mostraron que la edad, la educación y el accidente loxoscélico anterior son directamente proporcionales a los conocimientos de las participantes $(p<0,05)$. Conclusión: la mayoría de las mujeres encuestadas conocía la araña marrón y en gran parte reconoció el daño causado por la picadura, pero sobrestimaran la gravedad de las lesiones. Paradójicamente, el conocimiento de los síntomas fue pequeño, lo que puede llevar al retraso del tratamiento para sí mismas o para sus hijos. El conocimiento sobre la araña estuvo directamente relacionado con la edad, la educación y el accidente loxoscélico anterior.
Palabras clave:

Aracnidismo

Mordeduras y Picaduras

de Insectos

Salud Pública

Prevención de Accidentes

Educación en Salud

\section{Introdução}

O envenenamento provocado pela picada de aranhas do gênero Loxosceles é chamado de loxoscelismo, doença mais comumente causadora de uma lesão que regride sem tratamentos específicos, e raramente uma síndrome necrotizante-hemolítica. ${ }^{1-3} \mathrm{O}$ loxoscelismo representa mais de $50 \%$ dos acidentes notificados anualmente por aracnídeos no Brasil, principalmente nos estados do Sul, onde responde sozinho por $44 \%$ destas notificações, sendo um importante tema para ser discutido dentro da Atenção Primária à Saúde (APS). 4,5 O número de acidentes loxoscélicos vem aumentando nas últimas décadas, o que está relacionado a fatores como o grande e crescente número de aranhas, devido principalmente ao processo de urbanização desordenado, moradias que favorecem sua instalação e proliferação, e ao desconhecimento da população com relação à aranha e às lesões por ela provocadas ${ }^{6-8} A$ frequência de picadas por Loxosceles, principalmente L. Intermedia, mostra correlação com a área da cidade, sendo que a maior parte é na zona urbana (74,4\%), atingindo mais pessoas entre 20 e 49 anos $(60,5 \%)$, com predomínio do sexo feminino $(61 \%) .^{9,10}$

Dentre os casos de loxoscelismo notificados, segundo estudo de Marques, em 33,9\% não foram registradas as circunstâncias acerca da picada, enquanto que 23,4\% das vítimas relataram não ter percebido onde ocorreu a picada; $14,4 \%$ estavam dormindo; $12,9 \%$ estavam dentro da casa; e 9,8\% estavam se vestindo. ${ }^{5}$ Quanto ao tratamento do loxoscelismo, constata-se maior efetividade da soroterapia para as formas cutâneas graves, quando realizada até 24 horas após o acidente, e maior eficácia da corticoterapia nas lesões leves ou iniciais quando feita até 48 horas após o acidente, justificando a importância da identificação precoce da lesão e a procura por auxílio médico. ${ }^{2,9} \mathrm{O}$ desenvolvimento de tratamentos mais efetivos é dificultado pelo fato de não haver um conhecimento adequado do mecanismo de ação do veneno, mas tratamentos novos estão em estudo e mostraram bons resultados in vitro, como o uso do eculizumab. ${ }^{11,12}$

As puérperas foram escolhidas como participantes deste estudo pelo fato de a mulher ser geralmente a principal responsável pelo ambiente domiciliar e cuidado dos filhos, e também pelo fato de a maior incidência de acidentes ocorrer entre mulheres, além de acidentes graves entre crianças..$^{9,11}$ O ambiente hospitalar, particularmente os leitos da maternidade, foi escolhido por prover maior número de participantes e facilitar a aplicação dos questionários. O objetivo deste estudo foi identificar o grau 
de conhecimento que puérperas possuem a respeito da Loxosceles, sua picada e conduta no caso de acidente com elas ou seus filhos.

\section{Métodos}

Foi realizado um estudo transversal quantitativo entre julho e outubro de 2011. O instrumento para coleta de dados foi um questionário estruturado com escala de mensuração quantitativa, preenchido pelos pesquisadores durante uma entrevista. O questionário continha 25 perguntas objetivas abrangendo perfil socioeconômico (faixa etária, escolaridade, renda familiar mensal, bairro, habitação), perguntas sobre a aranha-marrom (identificação, visualização no cotidiano, habitat) e sobre o loxoscelismo (identificação da lesão, contato direto com acidente loxoscélico e seguimento corporal acometido, situação propícia ao acidente, sazonalidade, gravidade, sintomatologia, complicações, conduta ao suspeitar do acidente, conhecimentos gerais sobre loxoscelismo). Imagens foram utilizadas nas questões que envolviam identificação da lesão e da aranha. Após a conclusão do questionário, todas as questões foram explicadas às participantes. Todas as participantes assinaram e receberam uma cópia do Termo de Consentimento Livre e Esclarecido (TCLE).

A população estudada foi do tipo não probabilística de voluntários, com um intervalo de confiança de $95 \%$, margem de erro de 5\% e um "n" de 208 participantes, das quais oito foram excluídas. Foram incluídas pacientes em puerpério imediato, considerado entre o primeiro e décimo dia do parto, internadas na maternidade do Hospital do Trabalhador em Curitiba-PR, entre julho e outubro de 2011, que concordaram em participar e assinaram o TCLE. Foram excluídos oito questionários preenchidos de forma incorreta ou incompleta. Os dados obtidos foram tabulados no programa Microsoft Excel $2007^{\circledR}$ e analisados pelos testes $\chi^{2}$ e teste Exato de Fisher. Este estudo foi aprovado pelo Comitê de Ética em Pesquisa do Hospital do Trabalhador sob o protocolo número CEP-SESA/HT 345/2011.

\section{Resultados}

Com relação aos dados obtidos no questionário, constatou-se que a faixa etária prevalente foi a de mulheres entre 19 e 25 anos (Gráfico 1), o grau de escolaridade mais prevalente foi o ensino médio completo/incompleto (Gráfico 2) e a renda média familiar mensal foi de 2,7 salários mínimos. De acordo com a distribuição geográfica, os bairros citados pelas entrevistadas foram divididos em dois grupos: periféricos (88\%) e centrais (12\%). O tipo de habitação mais prevalente foi o de casas de alvenaria $(64,5 \%)$.

Sobre o reconhecimento da aranha-marrom, as participantes foram questionadas se reconheceriam a aranha, e depois se solicitou que indicassem a imagem correta da aranha no questionário. A maioria (61\%) afirmou reconhecê-la e apontou a imagem correta, enquanto $12 \%$ apenas afirmaram reconhecê-la, e $27 \%$ não a reconheceram. As puérperas que afirmaram já ter visto a aranha-marrom em casa ou local de trabalho somaram $64 \%$, e $36 \%$ afirmaram ter alguém próximo e/ou ter sido picada por aranha-marrom; destes, os segmentos corporais mais acometidos foram coxa e perna. ${ }^{2}$

Sobre o reconhecimento da lesão ocasionada pela aranha-marrom, as participantes foram questionadas se reconheceriam a lesão, e depois solicitadas que indicassem a imagem correta da lesão no questionário. A maioria declarou não reconhecê-la (67,5\%), enquanto $20,5 \%$ afirmaram reconhecê-la e apontaram a imagem correta; $12 \%$ apenas afirmaram reconhecer a lesão. 


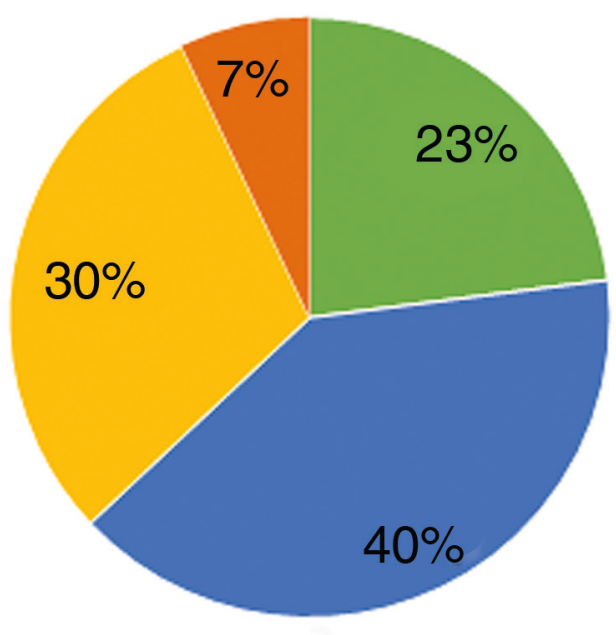

- 13 a 18 anos $(n=46)$

- 19 a 25 anos $(n=80)$

- 26 a 35 anos $(n=60)$

$\mathrm{n}=200$

$\because 36$ a 45 anos $(n=14)$

Gráfico 1. Idade das participantes.

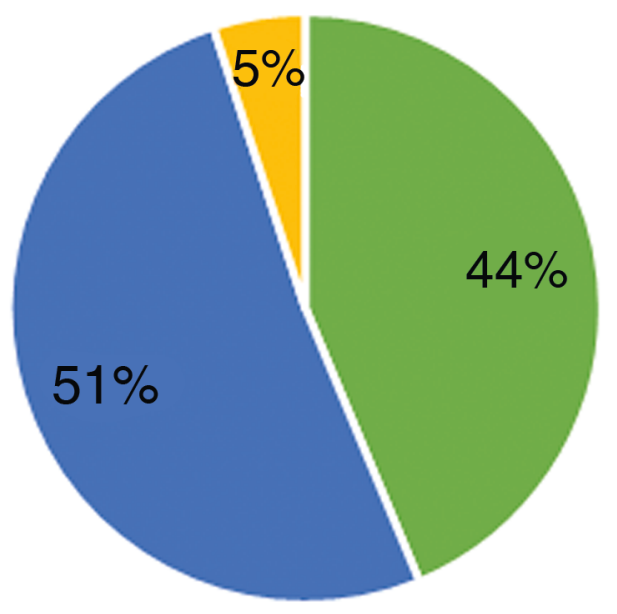

- Ensino fundamental completo/in completo $(n=88)$

- Ensino médio completo/incompleto ( $n=102)$

- Ensino superior completo/incompleto $(n=10)$ $\mathrm{n}=200$

Gráfico 2. Escolaridade das participantes. 
Quanto ao local mais provável para encontrar uma aranha-marrom, a maioria $(86,5 \%)$ respondeu corretamente que este local é atrás de quadros e armários. Quanto à situação que envolve o acidente Ioxoscélico, o maior grupo (79\%) respondeu corretamente que é mais comum durante a troca de roupa. Sobre a relação entre e a incidência do acidente loxoscélico, a época do ano e a temperatura, o maior grupo $(61 \%)$ respondeu corretamente que ocorrem mais acidentes nos meses mais quentes do ano. Quanto à gravidade do acidente loxoscélico, o maior grupo (78\%) respondeu incorretamente que ela é sempre grave.

Sobre a sintomatologia mais provável no loxoscelismo, apenas $37 \%$ responderam corretamente "dor, queimação e vômito". Quanto às complicações prováveis, apenas 36\% responderam corretamente "perda tecidual e insuficiência renal". Quanto à conduta ao suspeitar de acidente loxoscélico, o maior grupo $(87,5 \%)$ respondeu corretamente "capturar a aranha e procurar ajuda médica".

Por meio da estratificação da população estudada em grupos segundo o perfil obtido com o questionário, puderam-se comparar fatores que possuem relação positiva ou negativa com o grau de conhecimento geral sobre a aranha-marrom e o loxoscelismo. A análise dos dados mostrou que quanto maior a faixa etária das entrevistadas, maiores as taxas de reconhecimento da aranha-marrom, de conhecimento sobre a situação provável que envolve o acidente e da conduta correta ao suspeitar-se da picada por aranha-marrom $(p<0,05)($ Gráfico 3).

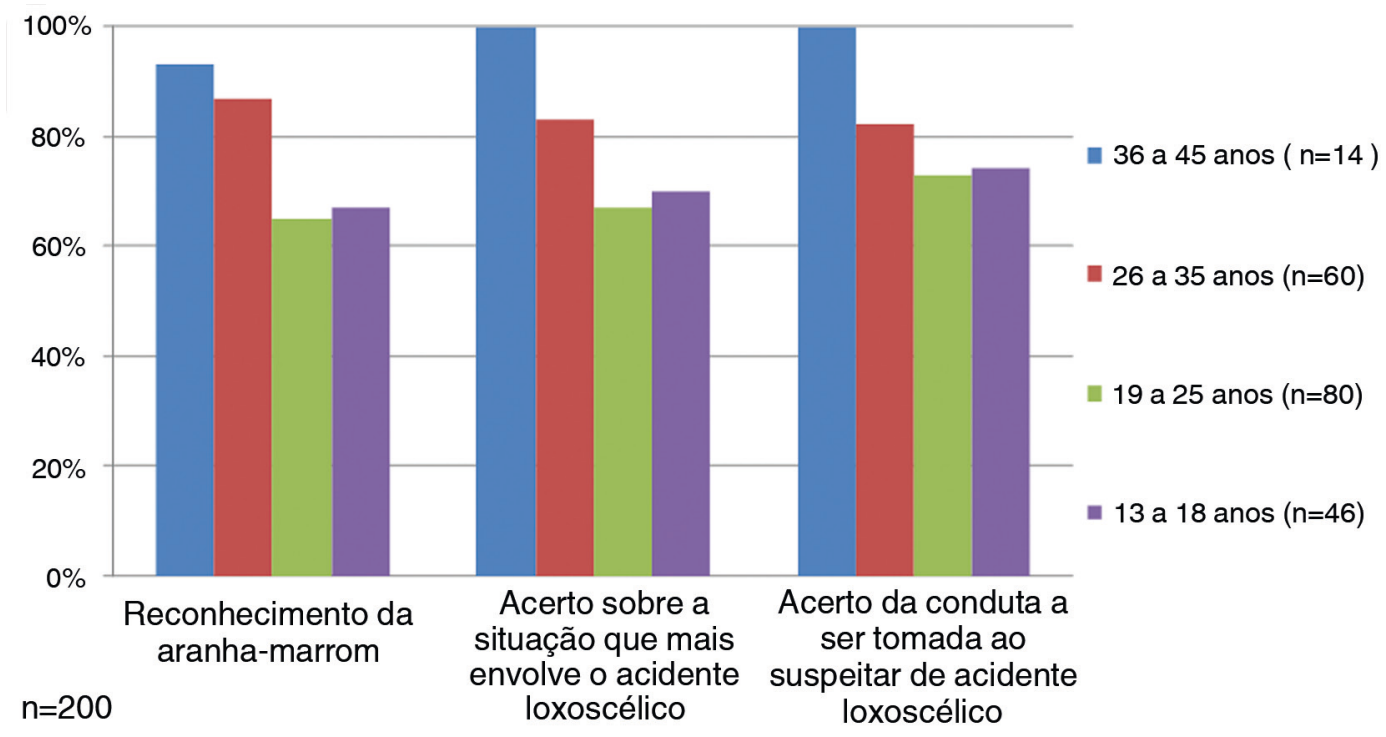

Gráfico 3. Respostas corretas sobre o reconhecimento da aranha-marrom, conhecimento sobre a situação que envolve o acidente e a conduta a ser tomada ao suspeitar de acidente loxoscélico, estratificadas por faixa etária em anos $(p<0,05)$.

A análise dos dados também mostrou uma relação positiva entre a escolaridade das participantes e as taxas de reconhecimento da aranha, de conduta correta ao suspeitar-se da picada e de conhecimentos gerais sobre o loxoscelismo $(p<0,05)$ (Gráfico 4).

Observou-se que quando a entrevistada reconheceu corretamente a imagem da aranha-marrom, suas taxas de acerto nas questões que abordavam o reconhecimento da lesão provocada pela aranha-marrom, habitat e situação provável que envolve o acidente loxoscélico foram maiores ( $p<0,05)$, conforme Gráfico 5. 


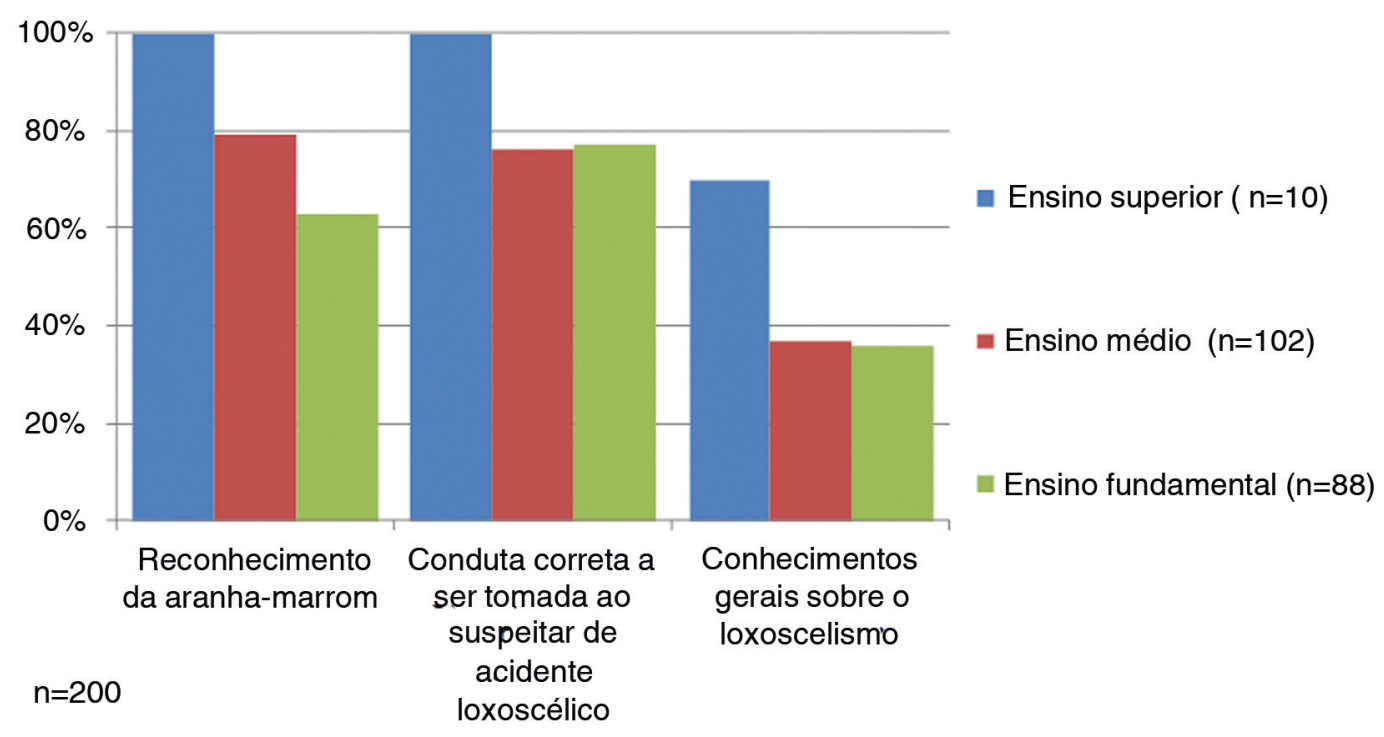

Gráfico 4. Respostas corretas sobre o reconhecimento da aranha-marrom, a conduta a ser tomada ao suspeitar de acidente loxoscélico e os conhecimentos gerais sobre o loxoscelismo, estratificadas por nível de graduação $(p<0,05)$.

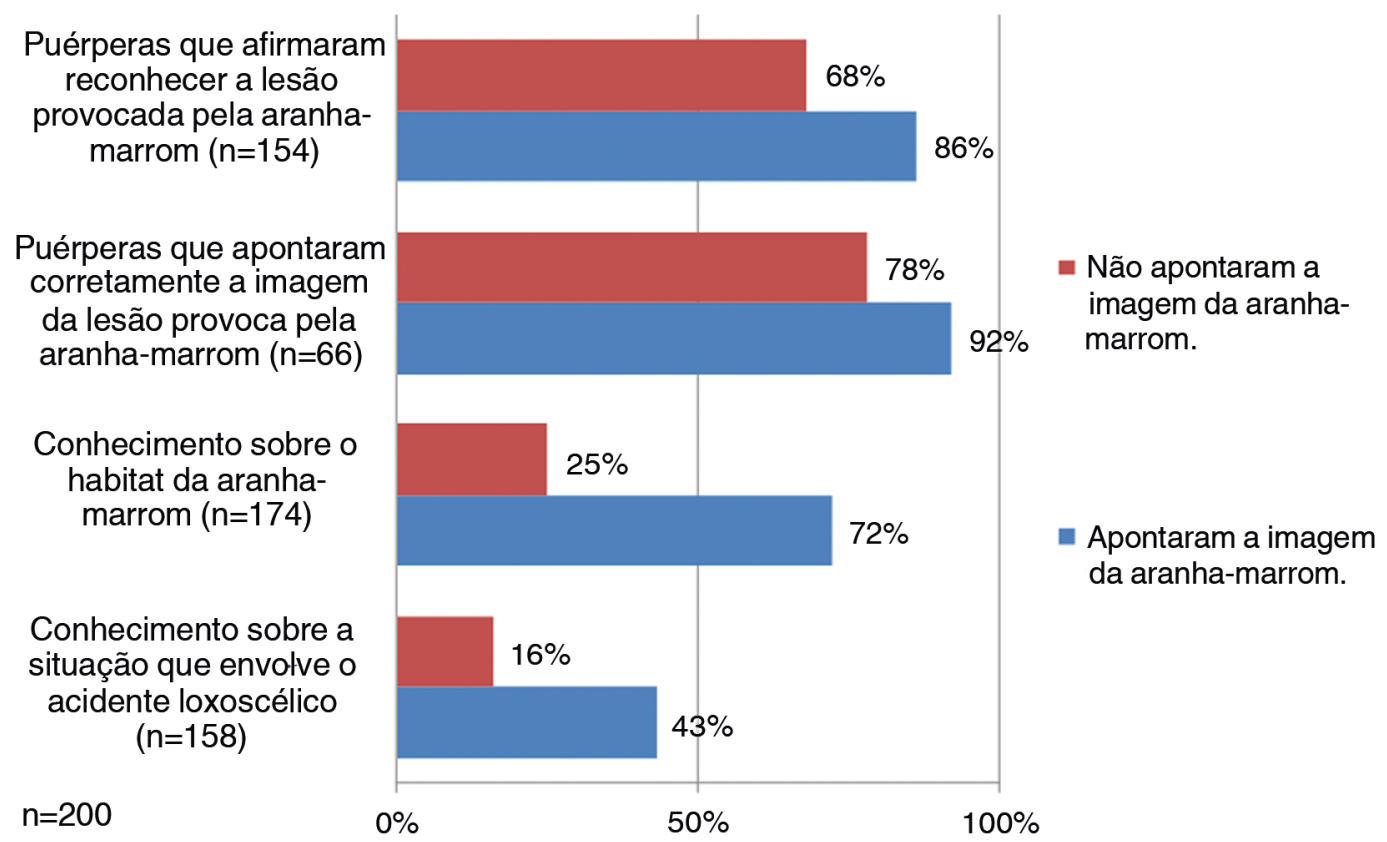

Gráfico 5. Respostas corretas sobre o reconhecimento da lesão provocada pela aranha-marrom, habitat e situação provável que envolve o acidente loxoscélico, estratificadas pelo reconhecimento correto versus não reconhecimento da imagem da aranha-marrom $(p<0,05)$.

Outra relação observada foi o fato de as entrevistadas que tiveram contato direto com o acidente loxoscélico (ter sofrido e/ou conhecer alguém que tenha sofrido o acidente) terem obtido taxas de acerto maiores nas questões que abordam o reconhecimento da aranha-marrom, terem visto uma aranha-marrom em casa ou local de trabalho e terem reconhecido a lesão provocada pela aranha-marrom - situação provável que envolve o acidente loxoscélico e conduta correta ao suspeitar-se da picada por aranha-marrom, quando comparadas às entrevistadas que nunca tiveram contato direto $(p<0,05)$. 


\section{Discussão}

O Sul do Brasil é a região que mais notifica acidentes por animais peçonhentos, sendo que dois terços são causados por aranhas do gênero Loxosceles. Metade desses acidentes ocorreu na região metropolitana de Curitiba (RMC), mantendo uma incidência de 150 casos para cada 100.000 habitantes. $^{2}$

O contato com a aranha-marrom nos domicílios da RMC é comum, ${ }^{1,2}$ o que se mostrou verdadeiro pelo fato de $33,5 \%$ das pesquisadas afirmarem que elas ou pessoas próximas já haviam tido algum contato com a aranha. A maioria das participantes afirmou conhecer a aranha e apontou a imagem correta no questionário, ocorrendo algo semelhante com a lesão provocada pela aranha, em que poucas afirmaram conhecê-la, mas $63 \%$ apontaram a imagem correta da lesão inicial no questionário. O presente estudo concordou com a literatura em relação ao segmento corporal mais acometido pela picada da aranha, que foi coxa e perna. ${ }^{2}$ A sazonalidade das aranhas é descrita na literatura e este estudo mostrou também que as participantes reconhecem essa particularidade. ${ }^{13}$

A maioria das participantes julgou o acidente loxoscélico invariavelmente grave, o que sugere um ponto de desconhecimento e trabalho em educação para as equipes das Unidades Básicas de Saúde ou de Estratégia Saúde da Família (ESF), já que são o primeiro local de atendimento e notificação das picadas. ${ }^{1,2} \mathrm{~A}$ maioria das lesões regride sem tratamento específico e requer apenas cuidados com a ferida ou pequenos desbridamentos de pele. ${ }^{3} \mathrm{O}$ acidente grave é incomum, e um estudo isolado mostrou maior incidência de hemólise mediada pela toxina de $L$. reclusa em crianças. ${ }^{11}$

Foi possível constatar um aumento progressivo de assertividade das participantes conforme o aumento da faixa etária nas questões sobre a identificação da aranha-marrom, situação mais provável para uma picada e sobre a primeira conduta ao suspeitar de uma picada $(p<0,05)$. Houve também um aumento percentual de questões corretas conforme o maior grau de escolaridade nas questões sobre identificação da aranha e a primeira conduta ao suspeitar de um acidente loxoscélico $(p<0,05)$. A literatura afirma que a educação sobre o loxoscelismo é uma importante ferramenta na prevenção de acidentes, e este trabalho concorda e sugere que a vivência das pacientes e a escolaridade também são importantes. ${ }^{6,14}$

A porcentagem de acertos das questões foi maior entre as participantes que já haviam sido picadas ou que tiveram alguém próximo que foi picado, nas questões sobre a identificação da aranha-marrom, lesão provocada, situação mais provável para picada e primeira conduta ao se suspeitar de um acidente $(p<0,05)$. Isso sugere que a experiência e/ou convivência influenciam no conhecimento destes assuntos, associado à maior preocupação sobre sua potencial gravidade.$^{5,15}$ Este estudo possui a limitação de ter sido feito com grupo específico de amostra não probabilística, estando sujeito ao viés de memória e de seleção.

\section{Conclusões}

A maioria das puérperas pesquisadas conhecia a aranha-marrom e grande parte reconhecia a lesão provocada pela picada, mas a gravidade da lesão foi superestimada pelas participantes da pesquisa. Contraditoriamente, o conhecimento dos sintomas provocados foi pequeno, o que pode levar ao atraso do tratamento delas ou dos seus filhos. Os resultados mostraram que o conhecimento da aranha está diretamente relacionado à idade, à escolaridade e a acidente loxoscélico prévio. 


\section{Referências}

1. Guimarães AB. Análise peptidômica comparativa das peçonhas de duas espécies de aranha marrom: Loxosceles laeta e Loxosceles intermedia [Dissertação de mestrado]. Brasília: Universidade de Brasília, Instituto de Química, Programa de Pós-Graduação;2009. Disponível em: http://repositorio.bce.unb.br/bitstream/10482/8874/1/2009_AlineBarbosaGuimaraes. pdf

2. Silva EM. Loxoscelismo no estado do Paraná: análise epidemiológica dos acidentes causados por Loxosceles Heinecken \& Lowe, 1832 no período de 1993 a 2000 [Dissertação de mestrado]. Rio de Janeiro: Fundação Oswaldo Cruz, Escola Nacional de Saúde Pública; 2002. Disponível em: http://www.saude.pr.gov.br/arquivos/File/zoonoses_intoxicacoes/ anim_pec/Loxoscelismo_no_Pr.pdf

3. Juckett G. Arthropod bites. Am Fam Physician. 2013;88(12):841-7. PMID: 24364549

4. Brasil. Ministério da Saúde. Acidentes por animais peçonhentos - Aranhas - Informações Técnicas. Disponível em: http:// portal.saude.gov.br/portal/saude/profissional/visualizar_texto.cfm?idtxt=39277

5. Silva EM, Souza Santos R, Fischer ML, Rubio GBG. Loxosceles spider bites in the state of Paraná, Brazil: 1993-2000. J Venom Anim Toxins Incl Trop Dis. 2006;12(1):110-23. DOI: http://dx.doi.org/10.1590/S1678-91992006000100009

6. Pinto LR, Mendonça F, Araujo WM. A influência das variações térmicas nos acidentes loxoscélicos em Curitiba/PR. Rev Bras Climatol. 2009;5:55-69.

7. Vetorello ML. A influência da temperatura, umidade relativa do ar e radiação solar na incidência dos acidentes loxoscélicos em Curitiba, Paraná, Brasil, no período de 1998 a 2001 [Dissertação de especialização]. Curitiba: Universidade Federal do Paraná, Setor de Ciências Biológicas, Departamento de Patologia; 2002.

8. Pinto LR. Loxoscelismo em Curitiba: uma abordagem geográfica [Dissertação de mestrado]. Curitiba: Universidade Federal do Paraná, Setor de Ciências da Terra, Departamento de Geografia; 2009.

9. Brasil. Secretaria Municipal de Saúde de Curitiba. Acidentes loxoscélicos - protocolo técnico e fluxo de atenção em Curitiba. Curitiba: Secretaria Municipal de Saúde; 2006 [Acesso 16 Ago 2015]. Disponível em: http://www.saude.curitiba.pr.gov.br/ images/institucional/arquivos/protocolos/protocolo_001.pdf

10. Trentini RP. Histórico do loxoscelismo e sua ocorrência no município de Curitiba - Paraná [Dissertação de especialização]. Curitiba: Universidade Federal do Paraná, Setor de Ciências da Terra, Programa de pós-graduação; 2002.

11. Gehrie EA, Nian H, Young PP. Brown Recluse spider bite mediated hemolysis: clinical features, a possible role for complement inhibitor therapy, and reduced RBC surface glycophorin A as a potential biomarker of venom exposure. PLoS One. 2013;8(9):e76558. Disponível em: http://dx.doi.org/10.1371/journal.pone.0076558

12. Tambourgi DV, Gonçalves-de-Andrade RM, van den Berg CW. Loxoscelism: From basic research to the proposal of new therapies. Toxicon. 2010;56(7):1113-9. DOI: http://dx.doi.org/10.1016/j.toxicon.2010.01.021

13. Rader RK, Stoecker WV, Malters JM, Marr MT, Dyer JA. Seasonality of brown recluse populations is reflected by numbers of brown recluse envenomations. Toxicon. 2012;60(1):1-3. DOI: http://dx.doi.org/10.1016/j.toxicon.2012.03.012

14. Buss PM. Promoção da saúde e qualidade de vida. Ciênc Saúde Colet. 2000;5(1):163-77. DOI: http://dx.doi.org/10.1590/ S1413-81232000000100014

15. Brasil. Ministério da Saúde, Fundação Nacional de Saúde. Manual de diagnóstico e tratamento de acidentes por animais peçonhentos. 2a ed. Brasília: Ministério da Saúde; 2001. 